\title{
Validation of the Pittsburgh esophageal perforation severity score: Further impetus for a prospective study
}

\author{
Mara B. Antonoff, MD
}

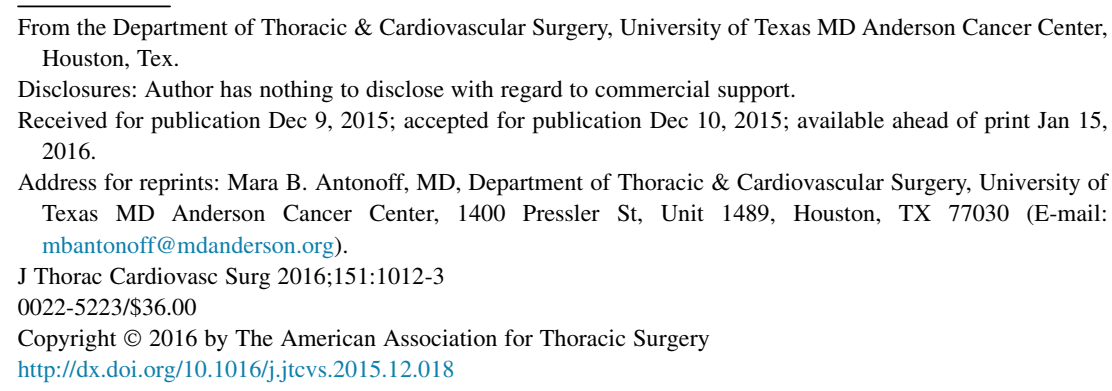

Esophageal perforation is widely recognized as a severely morbid condition, not infrequently met with unfavorable outcomes. ${ }^{1}$ This clinical problem has long been feared for its gravity, with historic recommendations favoring maximally invasive interventions. As innovative techniques have evolved and additional therapeutic options have become reasonable alternatives, strategies are clearly needed in order to allocate patients into appropriate treatment modalities.

In 2009, an important contribution was published out of the University of Pittsburgh, describing a novel perforation severity score (PSS) and hypothesizing that patients' optimal treatment strategies could be determined by quantification of the extent of mediastinal and pleural contamination. ${ }^{1}$ This study was met with great interest, as momentum in the field was building with experts questioning the dictum of determining operative strategy solely by the amount of time between perforation and presentation. While the Pittsburgh study included 119 patients in a single institution, external applicability remained to be assessed.

In the study by Schweigert and colleagues ${ }^{2}$ in this issue, the authors aimed to validate the Pittsburgh PSS system in an external cohort, applying the scale in a multinational setting over a 25-year period. The investigators retrospectively calculated the PSS for patients at 11 centers, predominantly European in location. In this important study, the authors divided the patients into 3 groups based on PSS, in accordance with the original Pittsburgh work (group 1: PSS $\leq 2$; group 2: PSS 3-5; and group 3: PSS > 5). Patients in group 3 displayed the worst outcomes, and, when compared with group 1, experienced 10 times the morbidity $(P<.0001)$ and 18 times the mortality $(P<.00001)$ of those patients with the lowest PSS. Furthermore, the authors found that patients with lower PSS were less frequently managed with operative intervention compared with patients with higher scores.

Overall, this article ${ }^{2}$ represents an excellent validation study, demonstrating the utility of the Pittsburgh scoring system in a different setting. The consistency of findings renders the PSS a reliable and useful tool; with these results,

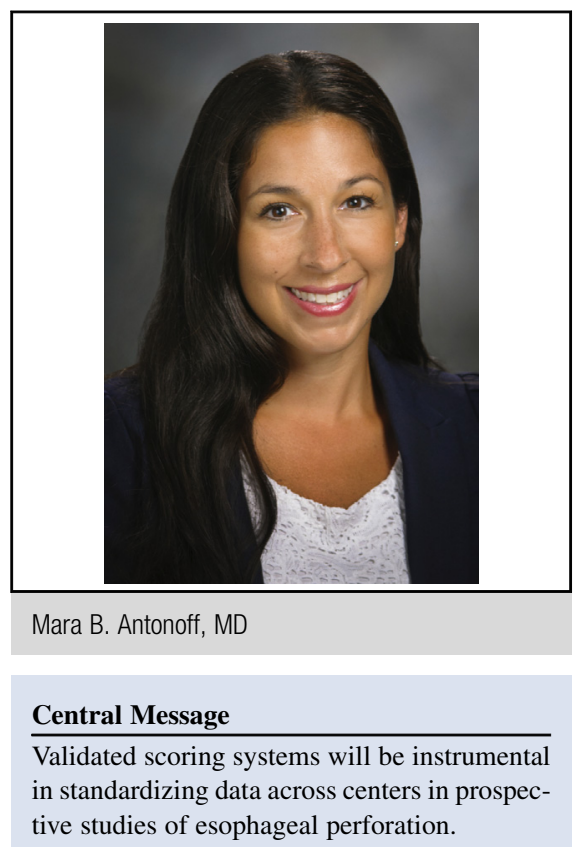

See Article page 1002.

efforts to apply the scoring system in a prospective manner are highly warranted. However, one ought to be careful about drawing any significant conclusions regarding optimal patient management based on these analyses alone. In their article, Schweigert and colleagues ${ }^{2}$ suggest that the Pittsburgh scoring system may identify patients suitable for nonoperative treatment, and, ultimately, an algorithm is proposed for selecting those patients who may avoid operative intervention. The authors retrospectively found less morbidity/mortality and less frequent operative management among patients in group 1, and thus, a recommendation was formulated favoring less invasive management for these individuals. The additional step of evaluating the success of nonoperative management in each group, either through further analyses of the current study or with future prospective studies, is needed in order to make such recommendations.

In reviewing these data, it is important to recognize that PSS was not used to determine treatment; rather, it was calculated retrospectively. For prospective evaluation in such an uncommon clinical entity, a multi-institutional study will clearly be needed. The present study effectively stratifies patients by risk for morbidity and mortality, and by likelihood of having received invasive treatment. 
However, without knowing the success of nonoperative versus operative treatment in each group, one must thoughtfully consider putting substantial weight into the suggested algorithm. It clearly makes sense that those individuals with likelihood of the worst outcome should receive the most aggressive care, but, scientifically, it should be demonstrated that more invasive treatment results in improved outcomes for those with higher scores. For patients with sepsis, it is obvious that source control is needed to improve survival; however, as our repertoire of available interventions expands in the realms of endoscopy and image-guided procedures, it is not as clear when maximally invasive strategies are absolutely mandatory. Moreover, as minimally invasive surgical procedures start to have more overlap with the procedures that are performed in gastrointestinal endoscopy and interventional radiology suites, the lines become more blurred. With goals of achieving wide drainage and enteral access, to what extent does the exact means make a difference? Certainly, there will always be a time and a place for open surgery; we must continue to engage in prospective studies evaluating the comparative efficacy of all strategies available, evolving our investigations as our therapeutic options expand.
In the present study, ${ }^{2}$ all participating institutions were esophageal centers. However, one might note that, over 25 years, the study included 288 patients at 11 locations, amounting to approximately 1 patient per year per center. This does not represent the same volume seen in the original work out of Pittsburgh, which averaged about 12 patients per year at a single institution. Nonetheless, the outcomes were quite similar, and the scoring system upheld well. As we look toward future prospective studies, it will be of interest to investigate the influence of center volume on outcomes.

Schweigert and colleagues ${ }^{2}$ ought to be commended for this excellent contribution, which is highly relevant to the modern practice of thoracic surgery. Further demonstrating the utility of the Pittsburgh esophageal PSS, this study supports the notion that prospective, large-scale studies are in need, and that such scoring systems will be instrumental in standardizing data across centers.

\section{References}

1. Abbas G, Schuchert MJ, Pettiford BL, Pennathur A, Landreneau J, Landreneau J, et al. Contemporaneous management of esophageal perforation. Surgery. 2009; 146:749-55; discussion 755-6.

2. Schweigert M, Santos Sousa H, Solymosi N, Yankulov A, Jiminez Fernandez M Beattie R, et al. Spotlight on esophageal perforation: A multinational study using the Pittsburgh esophageal perforation severity scoring system. J Thorac Cardiovasc Surg. 2016;151:1002-11. 